\title{
Retraso en el diagnóstico de lepra como factor pronóstico de discapacidad en una cohorte de pacientes en Colombia, 2000-2010
}

\author{
Martha Inírida Guerrero, ${ }^{1}$ Sandra Muvdi ${ }^{1}$ y Clara Inés León ${ }^{1}$
}

Forma de citar

Guerrero MI, Muvdi S, León CI. Retraso en el diagnóstico de lepra como factor pronóstico de discapacidad en una cohorte de pacientes en Colombia, 2000-2010. Rev Panam Salud Publica. 2013;33(2):137-43.

RESUMEN Objetivo. Evaluar los factores pronósticos de la presencia de discapacidad al momento del diagnóstico de lepra en una cohorte de pacientes colombianos de 2000 a 2010.

Métodos. Estudio analítico y observacional descriptivo de una cohorte retrospectiva de pacientes ingresados con diagnóstico de lepra en el Centro Dermatológico Federico Lleras Acosta, de Bogotá, Colombia, entre 2000 y 2010. Se realizó el análisis descriptivo de las variables y se identificaron factores pronósticos de la presencia de discapacidad al momento del diagnóstico mediante análisis simple y multifactorial (modelo de riesgos proporcionales de Cox); se calcularon las razones de riesgo (hazard ratio) para cada uno de los factores incluidos en el modelo.

Resultados. El tiempo entre los primeros síntomas y el diagnóstico en los 333 pacientes de la cohorte fue en promedio 2,9 años; 32,3\% de ellos tenían algún grado de discapacidad, especialmente en los pies. Hubo una mayor proporción de retraso en el diagnóstico y discapacidad en hombres que en mujeres y en pacientes con lepra multibacilar que con paucibacilar. La discapacidad se asoció significativamente con demoras $\geq 1$ año en el diagnóstico, edad $\geq 30$ años, indice baciloscópico inicial $\geq 2$, lepra multibacilar y proceder de Cundinamarca o Santander. Los factores protectores fueron ser del sexo femenino, tener algún grado de escolaridad y residir en Boyacá.

Conclusiones. El tiempo entre los primeros síntomas y el diagnóstico constituye el factor pronóstico clave de la discapacidad al momento del diagnóstico de lepra. Se recomienda reforzar la búsqueda activa de personas infectadas y promover el diagnóstico precoz.

Palabras clave Lepra; factores de riesgo; diagnóstico tardío; personas con discapacidad; Colombia.

La lepra, una enfermedad infecciosa crónica causada por el bacilo Mycobacterium leprae, provoca daños en la función nerviosa que pueden llegar a ser permanentes si no se detecta y trata oportunamente (1-3). Tanto desde el punto de vista económico como social y humano,

\footnotetext{
1 Instituto Nacional de Dermatología, Centro Dermatológico Federico Lleras Acosta, Bogotá, D.C., Colombia. La correspondencia se debe dirigir a: Martha Inírida Guerrero, correo electrónico: marthainiridag@yahoo.com
}

la discapacidad es la consecuencia más grave de la lepra $\mathrm{y}$, por lo tanto, a la que más atención se debe dedicar a fin de prevenirla. Por ello, la Organización Mundial de la Salud (OMS) propuso prestar mayor atención a la evaluación de estas secuelas al momento del diagnóstico de la lepra, de modo que se puedan identificar oportunamente los pacientes con discapacidad - $\mathrm{O}$ en riesgo de desarrollarla- y ofrecerles el tratamiento adecuado a tiempo (4).
La tasa de discapacidad grado 2 (avanzada o visible) al momento del diagnóstico ha permanecido estable en el mundo en los últimos 10 años, con valores de 0,25 a 0,29 por 100000 habitantes (5). En 2010, la proporción de casos con discapacidad al momento del diagnóstico mostraba una gran heterogeneidad geográfica, que iba desde cero en las Islas Marshall hasta 22,8\% en Sudán (5). En la Región de las Américas, Bolivia informó la menor proporción, con $3,2 \%$, y Para- 
guay la mayor con $13,0 \%$ (6). Se ha recomendado utilizar este indicador para evaluar los resultados de los programas de control (1), sin embargo, como en realidad solo permite cuantificar la captación de enfermos en estadios avanzados de la enfermedad, se debe tomar como un indicador tardío.

En Colombia, 9,80\% de los casos nuevos presentaban discapacidad grado 2 en 2007, la tasa más alta en la Región de las Américas ese año (7), y se mantuvo elevada en $2009(7,48 \%)$ (5) y 2010 $(9,20 \%)(6)$.

Toda vez que el retraso en la detección de los pacientes con lepra es un factor de riesgo importante para el desarrollo de la discapacidad, los programas de control en muchos países dan una alta prioridad a la detección temprana y se ha trabajado intensamente en la búsqueda de una herramienta que permita predecir el grado de discapacidad que se generaría según los diferentes tiempos de retraso en el diagnóstico (8). De hecho, se ha encontrado una asociación estadísticamente significativa entre el retraso mayor de un año en el diagnóstico, por una parte, y la clasificación del tipo de lepra como multibacilar, la forma clínica lepromatosa (9) y la presencia de discapacidad en el momento del diagnóstico, por otra (9, 10). Van Veen y colaboradores demostraron que la proporción de casos con discapacidad se incrementó a $67 \%$ cuando el retraso en el diagnóstico era de 3 a 4 años y aumentaba hasta $81 \%$ cuando el retraso era de más de 4 años (8).

El elevado número de casos con discapacidad grado 2 al momento del diagnóstico en el mundo sugiere que los esfuerzos realizados para lograr la detección temprana de los casos de lepra - que constituye el objetivo táctico central para eliminar esta enfermedad- aún no son suficientes (5). Lo más preocupante es que esta situación afecta tanto a los países donde la lepra sigue siendo un problema de salud pública como a los que ya han eliminado la enfermedad, donde se pueden revertir los avances logrados.

El Centro Dermatológico Federico Lleras Acosta (CDFLLA), de Bogotá, Colombia, es una institución especializada en enfermedades dermatológicas que atiende pacientes provenientes de cualquier parte del país, ya sea por demanda espontánea o por remisión de otras instituciones de salud de menor complejidad.
Teniendo en cuenta los antecedentes mencionados y la ausencia de datos nacionales al respecto, el objetivo del presente estudio fue evaluar los factores pronósticos de la presencia de discapacidad al momento del diagnóstico de lepra en una cohorte de pacientes colombianos atendidos en el CDFLLA en el período de 2000 a 2010.

\section{MATERIALES Y MÉTODOS}

Se desarrolló un estudio analítico y observacional descriptivo en una cohorte retrospectiva de los pacientes ingresados en el CDFLLA con diagnóstico de lepra entre enero de 2000 y diciembre de 2010.

El diagnóstico de lepra de los pacientes de esta cohorte se realizó según los criterios de la Guía de Atención Integral en Lepra, del Ministerio de la Protección Social de Colombia (11). Brevemente: los pacientes que ingresan al programa del CDFLLA reciben polifarmacoterapia, sesiones de fisioterapia y evaluaciones clínicas y bacteriológicas periódicas hasta su egreso del programa cuando, después de un período de vigilancia postratamiento, se haya confirmado la curación según criterios clínicos y bacteriológicos. La evaluación neurológica estuvo a cargo de especialistas, mientras un fisioterapeuta determinó el grado de discapacidad en ambos ojos, manos y pies, desde grado 0 (no hay discapacidad) al grado 2 (avanzada o visible), según la clasificación de la OMS recogida en la guía colombiana (11).

Para este estudio se utilizó una base de datos, creada con el paquete informático Access 2007, para almacenar y procesar la información de los pacientes. Los datos clínicos (forma clínica, tipo epidemiológico, y grado de discapacidad e índice bacilar al momento del diagnóstico), sociodemográficos (sexo, edad al momento del diagnóstico, lugar de residencia, escolaridad y ocupación) y epidemiológicos (clasificación epidemiológica, lugar y método de captación y posible fuente de contagio) se tomaron de las historias clínicas de los pacientes; el tiempo transcurrido desde la aparición de los primeros síntomas hasta el inicio del tratamiento se obtuvo del paciente. La escolaridad se catalogó como "baja" cuando no se terminaron los estudios primarios y "algún grado" cuando al menos se completó la primaria.
Se definió como retraso en el diagnóstico si había trascurrido 1 año o más entre la presentación de los primeros síntomas y el diagnóstico de la enfermedad, que se correspondió con el momento del inicio del tratamiento. Se consideró que no hubo retraso en el diagnóstico si este demoró menos de un año, ya que según la literatura especializada, es baja la probabilidad de que en este lapso de tiempo se desarrolle algún tipo de discapacidad a causa de la enfermedad no tratada (8-10).

Se realizó el análisis descriptivo de las variables y se evaluó la fuerza de la asociación entre los factores pronósticos y la presencia de discapacidad al momento del diagnóstico, tanto mediante análisis simple como multifactorial. Se utilizó también el modelo de riesgos proporcionales de Cox y se calcularon las razones de riesgo (hazard ratio) para cada uno de los factores incluidos en el modelo, con ayuda de los programas Epi-Info $7.0 \mathrm{y}$ Stata 9; se utilizó un nivel de significación de 0,05 .

Esta investigación recibió el aval del Comité Científico del Instituto Nacional de Dermatología de Colombia; los datos de los pacientes se manejaron en forma anónima.

\section{RESULTADOS}

En el período de estudio, 333 pacientes recibieron el diagnóstico de lepra e ingresaron en el programa del CDFLLA. Las características clínicas y sociodemográficas de la cohorte de estudio se presentan en el cuadro 1.

La edad promedio de los pacientes al momento del diagnóstico fue de 49,1 años (mediana: 48 años; mínimo: 6; máximo: 85). Cerca de 70\% (IC95\%: 64,9 a 74,7$)$ de los pacientes tenían entre 16 y 60 años de edad cuando recibieron el diagnóstico; precisamente este grupo conforma la población económicamente activa en Colombia (cuadro 1).

La distribución de la edad al momento del diagnóstico por tipo de lepra, según la clasificación de la OMS, muestra que 67,4\% (IC95\%: 61,2 a 73,2) de los enfermos en edad económicamente activa presentó el tipo multibacilar, que es el más infectante y, por lo tanto, más importante desde el punto de vista epidemiológico por su alta capacidad de transmisión (cuadro 2). 
CUADRO 1. Características de la población de estudio, Centro Dermatológico Federico Lleras Acosta, Bogotá, Colombia, 2000-2010

\begin{tabular}{|c|c|c|c|}
\hline \multirow[t]{2}{*}{ Características } & \multirow[t]{2}{*}{ Casos } & \multicolumn{2}{|c|}{$\%(\operatorname{IC} 95 \%)^{\mathrm{a}}$} \\
\hline & & & \\
\hline Masculino & 212 & 63,7 & $(58,4-68,7)$ \\
\hline Femenino & 121 & 36,3 & $(31,3-41,6)$ \\
\hline \multicolumn{4}{|l|}{ Edad al diagnóstico (años) } \\
\hline $0-15$ & 4 & 1,2 & $(0,4-2,9)$ \\
\hline $16-30$ & 53 & 15,9 & $(12,3-20,1)$ \\
\hline $31-45$ & 93 & 27,9 & $(23,3-32,9)$ \\
\hline $46-60$ & 87 & 26,1 & $(21,6-31,1)$ \\
\hline $61-75$ & 74 & 22,2 & $(18,0-26,9)$ \\
\hline$>75$ & 22 & 6,6 & $(4,3-9,7)$ \\
\hline \multicolumn{4}{|l|}{ Procedencia } \\
\hline Cundinamarca & 121 & 36,4 & $(31,3-41,6)$ \\
\hline Santander & 50 & 15,0 & $(11,5-19,2)$ \\
\hline Tolima & 46 & 13,8 & $(10,4-17,8)$ \\
\hline Boyacá & 42 & 12,6 & $(9,4-16,5)$ \\
\hline Otros departamentos & 74 & 22,2 & $(18,0-26,9)$ \\
\hline \multicolumn{4}{|l|}{ Tipo epidemiológico } \\
\hline Multibacilar & 226 & 67,9 & $(62,7-72,7)$ \\
\hline Paucibacilar & 107 & 32,1 & $(27,3-37,3)$ \\
\hline \multicolumn{4}{|l|}{ Forma clínica } \\
\hline Indeterminada & 20 & 6,0 & $(3,8-8,9)$ \\
\hline Tuberculoide dimorfa & 18 & 5,4 & $(3,3-8,3)$ \\
\hline Lepromatosa dimorfa & 18 & 5,4 & $(3,3-8,3)$ \\
\hline Dimorfa-dimorfa & 58 & 17,4 & $(13,6-21,8)$ \\
\hline Tuberculoide & 48 & 14,4 & $(10,9-18,5)$ \\
\hline Lepromatosa & 145 & 43,6 & $(38,3-48,9)$ \\
\hline Neural pura & 26 & 7,8 & $(5,3-11,1)$ \\
\hline \multicolumn{4}{|l|}{ Discapacidad al momento del diagnóstico } \\
\hline Grado 0 & 211 & 63,4 & $(58,1-68,4)$ \\
\hline Grado 1 & 66 & 19,8 & $(15,8-24,4)$ \\
\hline Grado 2 & 51 & 15,3 & $(11,7-19,5)$ \\
\hline Sin dato & 5 & 1,5 & $(0,6-3,3)$ \\
\hline \multicolumn{4}{|l|}{ Índice bacilar al momento del diagnóstico } \\
\hline Multibacilar, 0 & 41 & 12,3 & $(9,1-16,2)$ \\
\hline Multibacilar, de 0,1 a 1,0 & 44 & 13,2 & $(9,9-17,2)$ \\
\hline Multibacilar, de 1,1 a 1,9 & 39 & 11,7 & $(8,6-15,5)$ \\
\hline Multibacilar, de 2,0 a 3,0 & 102 & 30,6 & $(25,9-35,7)$ \\
\hline Paucibacilar, 0 & 107 & 32,2 & $(27,3-37,3)$ \\
\hline \multicolumn{4}{|c|}{ Tiempo desde el inicio de los síntomas hasta el diagnóstico (años) } \\
\hline$<1$ & 107 & 32,1 & $(27,3-37,3)$ \\
\hline$\geq 1 \mathrm{y} \leq 3$ & 118 & 35,5 & $(30,4-40,7)$ \\
\hline$>3 y \leq 10$ & 62 & 18,6 & $(14,7-23,1)$ \\
\hline$>10$ & 18 & 5,4 & $(3,3-8,3)$ \\
\hline Sin dato & 28 & 8,4 & $(5,8-11,8)$ \\
\hline
\end{tabular}

a IC95\%: intervalo de confianza de $95 \%$.

CUADRO 2. Características de la población de estudio según la edad al momento del diagnóstico y el tipo de lepra, Centro Dermatológico Federico Lleras Acosta, Bogotá, Colombia, 2000-2010

\begin{tabular}{|c|c|c|c|c|c|c|}
\hline \multirow{3}{*}{$\begin{array}{l}\text { Edad al diagnóstico } \\
\text { (años) }\end{array}$} & \multicolumn{6}{|c|}{ Tipo epidemiológico } \\
\hline & \multicolumn{3}{|c|}{ Multibacilar } & \multicolumn{3}{|c|}{ Paucibacilar } \\
\hline & No. & $\%^{a}$ & IC95\% ${ }^{b}$ & No. & $\%^{a}$ & IC $95 \%$ b \\
\hline $0-15$ & 1 & 25,0 & $1,2-75,8$ & 3 & 75,0 & $24,2-98,8$ \\
\hline $16-30$ & 36 & 67,9 & $54,5-79,4$ & 17 & 32,1 & $20,6-45,5$ \\
\hline $31-45$ & 61 & 65,6 & $55,5-74,7$ & 32 & 34,4 & $25,3-44,5$ \\
\hline $46-60$ & 60 & 69,0 & $58,7-78,0$ & 27 & 31,0 & $22,0-41,3$ \\
\hline $61-75$ & 51 & 68,9 & $57,7-78,7$ & 23 & 31,1 & $21,3-42,3$ \\
\hline$>75$ & 17 & 77,3 & $56,6-91,2$ & 5 & 22,7 & $8,8-43,4$ \\
\hline Total & 226 & 67,9 & $62,7-72,7$ & 107 & 32,1 & $27,3-37,3$ \\
\hline
\end{tabular}

a Porcentajes de pacientes multibacilares y paucibacilares en cada grupo etario.

b IC95\%: intervalos de confianza de 95\%.
El tiempo promedio transcurrido entre la presentación de los primeros síntomas y el diagnóstico de la enfermedad fue de 2,9 años (moda: 2 años; mínimo 0; máximo: 50 ), aunque 28 casos no ofrecieron ese dato. En cuanto a la captación, $89,2 \%$ (IC95\%: 85,5 a 92,2) de los pacientes se presentaron espontáneamente en el CDFLLA, 10,4\% (IC95\%: 7,6 a 14,2) fueron resultado de la investigación de convivientes de casos diagnosticados y solo 0,3\% (IC95\%: 0,02 a 1,5) se captaron mediante la búsqueda activa de casos.

\section{Grado de discapacidad al momento del diagnóstico}

De los nuevos pacientes ingresados, 32,3\% (IC95\%: 26,7 a 38,3) presentaban algún grado de discapacidad al momento del diagnóstico; por cada paciente con discapacidad grado 2 se encontraron 1,6 pacientes con discapacidad grado 1 .

En general, la discapacidad más frecuentemente detectada fue en los pies, seguidos de las manos, y la menor proporción se observó en los ojos, sin diferencias entre el lado derecho y el izquierdo (figura 1). Al momento del diagnóstico, hubo menos mujeres con discapacidad que hombres (cuadro 3).

La relación entre el retraso en el diagnóstico y la presencia de discapacidad al momento del diagnóstico fue mayor en los hombres que en las mujeres, y en los pacientes con lepra del tipo multibacilar que en los pacientes con el tipo paucibacilar, posiblemente debido a un mayor retraso en el diagnóstico de estos últimos (figura 2).

\section{Análisis de los factores asociados}

Según el análisis bifactorial, la presencia de discapacidad en el momento del diagnóstico asociada significativamente con el retraso mayor de un año en el diagnóstico fueron: tener lepra del tipo multibacilar y la forma clínica lepromatosa, el diagnóstico con índices bacilares $\geq 2$, pertenecer al sexo masculino, tener 30 años de edad o más y poseer un bajo grado de escolaridad (cuadro 3).

En el análisis multifactorial se confirmó que las variables asociadas finalmente con una mayor posibilidad de presentar discapacidad al momento del ingreso al programa fueron: el retraso mayor de un año en el diagnóstico, te- 
FIGURA 1. Casos de discapacidad al momento del diagnóstico en pacientes con lepra, según su localización y sexo, Centro Dermatológico Federico Lleras Acosta, Bogotá, Colombia, 2000-2010

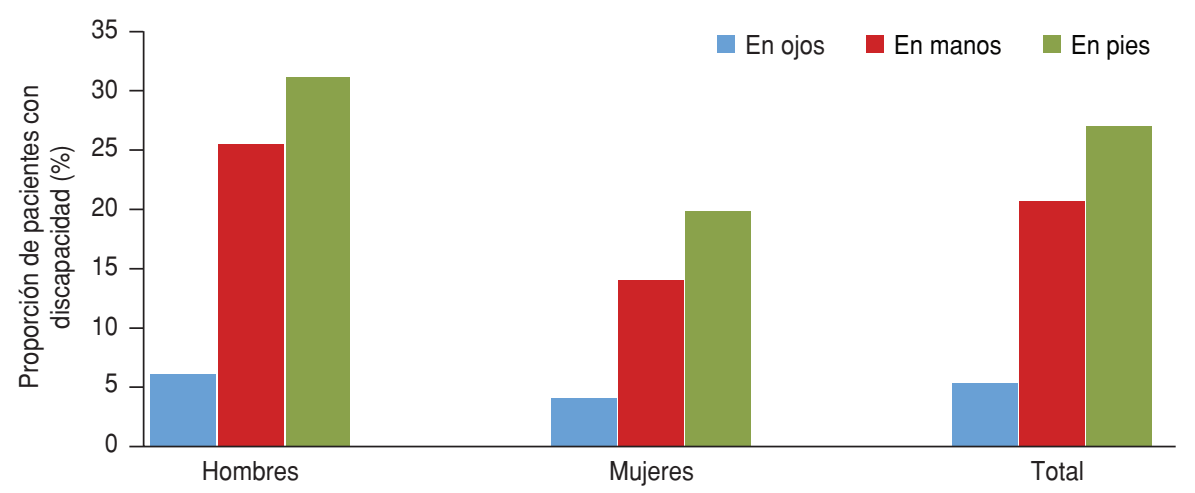

CUADRO 3. Análisis de los factores pronósticos de la presencia de discapacidad al momento del diagnóstico de lepra, Centro Dermatológico Federico Lleras Acosta, Bogotá, Colombia, 2000-2010

\begin{tabular}{|c|c|c|c|c|}
\hline Variable & $\begin{array}{c}\text { Casos con } \\
\text { discapacidad, (\%) }\end{array}$ & $\begin{array}{c}\text { Casos sin } \\
\text { discapacidad, (\%) }\end{array}$ & $\begin{array}{l}\text { Razón de riesgos, } \\
(\text { (IC95\%) }\end{array}$ & $P^{\mathrm{b}}$ \\
\hline \multicolumn{5}{|c|}{ Tiempo hasta el diagnóstico (años) } \\
\hline$\geq 1$ & $88(43,1)$ & $116(58,9)$ & \multirow{2}{*}{$2,0 \underset{\mathrm{c}}{(1,2-3,4)}$} & \multirow[t]{2}{*}{$<0,05$} \\
\hline$<1$ & $29(27,4)$ & $77(72,6)$ & & \\
\hline \multicolumn{5}{|l|}{ Tipo epidemiológico } \\
\hline Multibacilar & $92(41,4)$ & $130(58,6)$ & \multirow{2}{*}{$2,3 \underset{\mathrm{c}}{(1,4,3,9)}$} & \multirow[t]{2}{*}{$<0,05$} \\
\hline Paucibacilar & $25(36,4)$ & $82(76,6)$ & & \\
\hline \multicolumn{5}{|l|}{ Forma clínica } \\
\hline Lepromatosa & $61(43,3)$ & $80(56,7)$ & \multirow{3}{*}{$\underset{\mathrm{c}}{1,9}\left(\frac{1,1-3,1)}{4}\right.$} & \multirow[t]{3}{*}{$<0,05$} \\
\hline Dimorfa o indeterminada & $33(29,0)$ & $81(71,0)$ & & \\
\hline \multicolumn{3}{|l|}{ Índice bacilar inicial } & & \\
\hline$\geq 2,0$ & $44(44,0)$ & $56(56,0)$ & \multirow{2}{*}{ 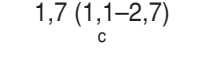 } & \multirow[t]{2}{*}{$<0,05$} \\
\hline$<2,0$ & $73(31,9)$ & $156(68,1)$ & & \\
\hline \multicolumn{5}{|l|}{ Sexo } \\
\hline Masculino & $84(40,4)$ & $124(59,6)$ & \multirow{2}{*}{$\underset{\mathrm{c}}{1,8} \underset{(1,1-2,9)}{ }$} & \multirow[t]{2}{*}{$<0,05$} \\
\hline Femenino & $33(27,3)$ & $88(72,7)$ & & \\
\hline \multicolumn{5}{|l|}{ Edad al diagnóstico (años) } \\
\hline$\geq 30$ & $105(37,9)$ & $172(62,1)$ & \multirow{3}{*}{$2,0 \underset{c}{(1,1-4,1)}$} & \multirow[t]{2}{*}{$<0,05$} \\
\hline$<30$ & $12(23,1)$ & $40(76,9)$ & & \\
\hline \multicolumn{4}{|l|}{ Escolaridad } & \\
\hline Baja & $86(38,4)$ & $138(61,6)$ & \multirow{2}{*}{$\underset{c}{1,8} \underset{c}{(1,1-3,1)}$} & \multirow[t]{2}{*}{$<0,05$} \\
\hline Algún grado & $22(25,9)$ & $63(74,1)$ & & \\
\hline
\end{tabular}

a IC95\%: intervalos de confianza de 95\%.

b $P$ : prueba de Fisher; nivel de significación $P<0,05$.

c Grupo de comparación o referencia.

FIGURA 2. Relación entre el retraso en el diagnóstico y la presencia de discapacidad al momento del diagnóstico de los pacientes según el sexo y el tipo de lepra, Centro Dermatológico Federico Lleras Acosta, Bogotá, Colombia, 2000-2010

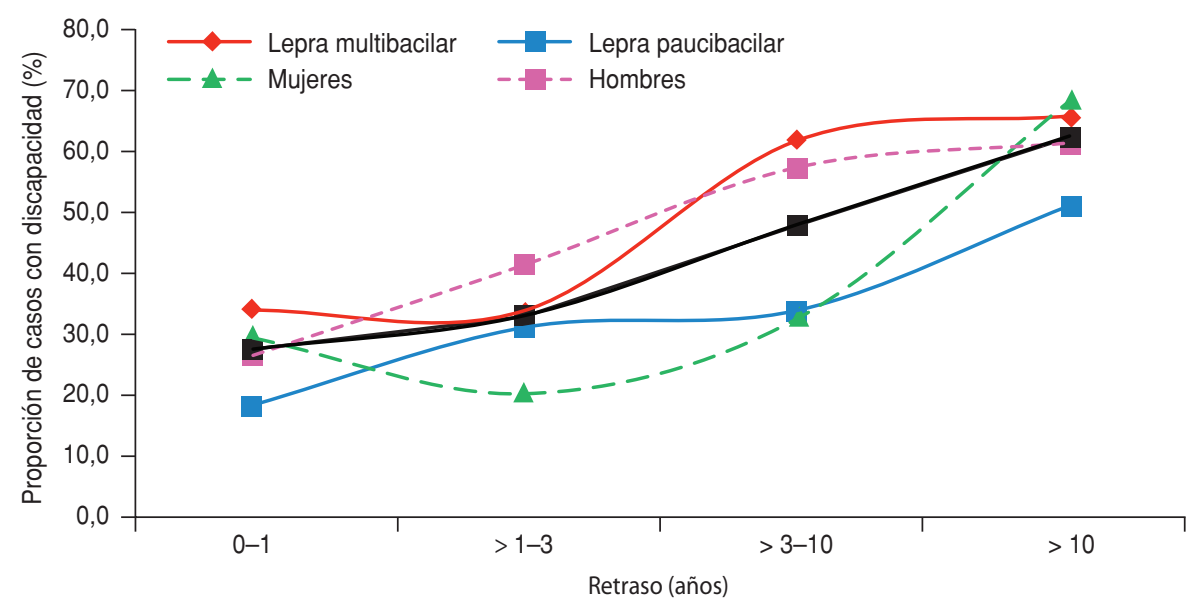

ner más de 30 años de edad, padecer el tipo multibacilar y presentar un índice bacilar inicial $\geq 2$. En cuanto al departamento de procedencia de los pacientes, se encontró una mayor razón de riesgos en los procedentes de Cundinamarca y, aunque en menor grado, de Santander. El mejor pronóstico (mayor posibilidad de no presentar discapacidad al momento del diagnóstico) lo tuvieron las mujeres y las personas con algún grado de escolaridad (cuadro 4). En cuanto al lugar de procedencia, los pacientes provenientes de Boyacá presentaron la menor razón de riesgos.

\section{DISCUSIÓN}

Según los resultados de este estudio, la población económicamente activa (de 16 a 60 años) es la más afectada por la lepra en Colombia, lo que implica un elevado costo social y económico al país, especialmente cuando provoca discapacidad, lesiones y reacciones lepróticas tipos 1 y 2, que alejan a estos pacientes de la actividad productiva y les causan trastornos en su vida social. Además, la alta proporción de enfermos con lepra del tipo multibacilar en este grupo de edad $(67,4 \%)$ sería una indicación de que la transmisión de la enfermedad puede ser elevada no solo en el entorno social y familiar, sino también en el entorno laboral en el que se desenvuelve la mayoría de estos pacientes $(12,13)$.

En esta cohorte, el tiempo de retraso entre la presentación de los primeros síntomas y el diagnóstico se movió en un espectro muy amplio, lo que puede explicarse por el hecho de que 89,2\% de los pacientes se presentaron espontáneamente en el CDFLLA en busca de diagnóstico, es decir, el Sistema de Seguridad Social no los buscó ni detectó. En este estudio se confirmó que existen deficiencias en la búsqueda activa de casos de lepra (14) y que faltan acciones que incentiven la pronta asistencia de los convivientes y otros contactos de los casos a los servicios de salud.

Solo $32,1 \%$ de los pacientes recibieron el diagnóstico de la enfermedad durante el primer año de la aparición de los síntomas. Aunque este lapso se considera apropiado para ingresar al tratamiento, hay también autores que sugieren limitar a seis meses el período adecuado para el diagnóstico, lo que incrementaría la sensibilidad de la medición (8) y reduciría la probabilidad de que la enferme- 
dad provoque alguna discapacidad; la proporción de pacientes diagnosticados después de este plazo podría utilizarse como un indicador operativo de deficiencias en la calidad de los servicios y del programa colombiano de control de lepra (9).

Al comparar estos resultados con los de un estudio realizado entre 1951 y 1999, en el que Buitrago y colaboradores evaluaron una población de la misma zona geográfica y características similares a las del presente estudio (15), se observa que el tiempo medio de retraso en el diagnóstico se redujo de 5,4 años a 2,9 años y que la proporción de los casos que presentaban algún grado de discapacidad en el momento del diagnóstico también disminuyó de 54,0\% (IC95\%: 48,3 a 59,6 ) a $32,3 \%$ (IC95\%: 26,7 a 38,3 ). Esta importante reducción de 22 puntos porcentuales en la presencia de discapacidad al momento del diagnóstico puede deberse al efecto positivo de los 11 años de funcionamiento del Programa de Control de Lepra en Colombia.

De los casos con discapacidad al momento del diagnóstico, 7,2\% (IC95\%: 4,4 a 10,9$)$ la tenía en los ojos, cifra similar al 8\% (IC95\%: 5,4 a 11,6) encontrado por Buitrago y colaboradores en 1999 (15) y a los valores de $4,6 \%$ a $11,0 \%$ encontra- dos en Etiopía (16), Nepal (17), e India, Filipinas y Etiopía (18). Sin embargo, los datos aquí presentados difieren de los de otro estudio realizado en 1999, también en pacientes del CDFLLA, en el que la prevalencia de lesiones oculares alcanzaba 58,9\% (IC95\%: 49,6 a 67,8); esta diferencia puede deberse a que esos pacientes ya estaban en tratamiento desde hacía varios años y los evaluó un especialista en oftalmología, que podía detectar daños menos visibles para un clínico (19). Ese estudio reveló la necesidad de que la evaluación de este tipo de discapacidad estuviera a cargo de un especialista o, en su defecto, lo realice personal no especializado, pero debidamente capacitado (19).

La frecuencia de la discapacidad en las manos al momento del diagnóstico fue de 18,7\% (IC95\%: 14,3 a 23,9), es decir, 2,5 veces menor que la informada por Buitrago y colaboradores (47,6\%; IC $95 \%$ : 42,0 a 53,3) (15). La discapacidad en los pies al momento del diagnóstico fue la más frecuente en esta cohorte $(25,5 \%)$, aunque representaba menos de la mitad de la informada en 1999 (15) y en un estudio similar realizado en Etiopía (20). Se observaron más casos de discapacidad en manos y pies (grados 1 y 2) en los hombres que en las mujeres $(P<0,05)$, posiblemente debido a que los hombres prestan menos atención a los síntomas.

Las variaciones encontradas en el riesgo de tener discapacidad al momento del diagnóstico de lepra en los pacientes de diferentes departamentos colombianos confirman que la frecuencia de estos casos varía entre los países y dentro de un mismo país. Por ejemplo, en Brasil, mientras en Bello Horizonte se informaba una frecuencia de discapacidad en manos y pies de 36,8\% (21), en Espirito Santo se encontró una frecuencia de solamente $6,0 \%$ (22). Las diferencias encontradas en este estudio entre los departamentos podrían explicarse por la mayor o menor disponibilidad de instituciones públicas prestadoras de salud, que tienen bajo su responsabilidad los programas locales de lepra (23). Así, en los pacientes procedentes de Cundinamarca y Santander, regiones colombianas con relativamente menos instituciones de este tipo, se observó un mayor riesgo de diagnóstico con algún grado de discapacidad que en los provenientes del departamento de Boyacá, donde hay una mayor oferta de servicios en su red pública (cuadro 5).

La proporción de casos nuevos diagnosticados con discapacidad constituye una medida indirecta de los casos no

CUADRO 4. Análisis multifactorial de los factores pronósticos de discapacidad al momento del diagnóstico de lepra, Centro Dermatológico Federico Lleras Acosta, Bogotá, Colombia, 2000-2010

\begin{tabular}{|c|c|c|c|c|c|c|}
\hline Factores & Razón de riesgos ${ }^{a}$ & IC95\% ${ }^{b}$ & Coeficiente & Error estándar & Estadístico $z$ & $P$ \\
\hline Tipo de lepra (paucibacilar vs. multibacilar ${ }^{\mathrm{c}}$ ) & 1,3635 & $1,294-1,4368$ & 0,3101 & 0,0267 & 11,6112 & $<0,001$ \\
\hline Índice bacilar inicial $\left(<2,0\right.$ vs. $\left.\geq 2,0^{\circ}\right)$ & 1,4855 & $1,4046-1,571$ & 0,3957 & 0,0285 & 13,8636 & $<0,001$ \\
\hline Departamento de procedencia (otro vs. Cundinamarca ${ }^{C}$ ) & 1,1086 & $1,0593-1,1601$ & 0,1031 & 0,0232 & 4,4449 & $<0,001$ \\
\hline Escolaridad (baja vs. algún gradod) & 0,8012 & $0,761-0,8434$ & $-0,2217$ & 0,0262 & $-8,4484$ & $<0,001$ \\
\hline Edad $\left(<30\right.$ años $v s . \geq 30$ años $\left.^{c}\right)$ & 1,3022 & $1,2097-1,4018$ & 0,2641 & 0,0376 & 7,0201 & $<0,001$ \\
\hline Sexo (masculino vs. femenino ${ }^{d}$ ) & 0,8883 & $0,8474-0,931$ & $-0,1185$ & 0,024 & $-4,9366$ & $<0,001$ \\
\hline
\end{tabular}

a Según el análisis de riesgos proporcionales de Cox.

b IC95\%: intervalo de confianza de 95\%.

c Factor pronóstico.

d Factor protector.

CUADRO 5. Descripción de la capacidad instalada en la red pública de prestación de servicios de salud en algunos departamentos de Colombia, 2010

\begin{tabular}{|c|c|c|c|c|c|c|c|c|c|c|c|}
\hline \multirow[b]{2}{*}{ Departamento } & \multirow[b]{2}{*}{ Población } & \multirow[b]{2}{*}{ Municipios } & \multicolumn{3}{|c|}{ IPS, ${ }^{a}$ por nivel } & \multicolumn{3}{|c|}{$\begin{array}{c}\text { Consultorios de atención } \\
\text { externa, por nivel }\end{array}$} & \multirow{2}{*}{$\begin{array}{l}\text { IPSa por } \\
\text { municipio }\end{array}$} & \multirow{2}{*}{$\begin{array}{c}\text { IPS }{ }^{a} \text { por } \\
10000 \text { habitantes }\end{array}$} & \multirow{2}{*}{$\begin{array}{c}\text { Profesionales } \\
\text { de la salud por } \\
10000 \text { habitantes }\end{array}$} \\
\hline & & & I & II & III & I & II & III & & & \\
\hline Boyacá & 1267597 & 123 & 104 & 11 & 1 & 347 & 11 & 15 & 0,94 & 0,915 & 9 \\
\hline Cundinamarca & 2477036 & 116 & 44 & 9 & 2 & 326 & 175 & 67 & 0,47 & 0,222 & 7 \\
\hline Santander & 2010404 & 87 & 80 & 11 & 4 & 317 & 48 & 30 & 1,09 & 0,472 & 5 \\
\hline
\end{tabular}

Fuente: Elaborado a partir de datos de la referencia 23

a IPS: número de instituciones prestadoras de salud. 
diagnosticados presentes en una población (24), por lo que los resultados de este estudio confirman que en Colombia hay una prevalencia de lepra mayor de la registrada. Cuevas y colaboradores encontraron que la prevalencia real de lepra en 10 municipios colombianos en el período 2002-2004 era entre 2 y 10 veces mayor que la recogida en los registros epidemiológicos de esos municipios (25).

A pesar de que la OMS recomienda monitorear solo los casos con discapacidad grado 2 (4), es importante prestar atención también a los casos de discapacidad grado 1 para tener una mejor idea de la prevalencia oculta y para que un mayor número de pacientes pueda recibir tratamiento oportuno y resolver su aún incipiente discapacidad. En un estudio realizado entre 1993 y 2003 en Bello Horizonte, Brasil, se encontró que $43,2 \%$ de las discapacidades grado 1 se podían revertir, mientras que solo $21,3 \%$ de las discapacidades grado 2 se revertían; además, 20\% de las discapacidades grado 2 pueden mejorar y pasar a discapacidad grado 1 (21). Todo esto respalda la importancia y la necesidad de fortalecer las acciones y las políticas dirigidas a apoyar el diagnóstico oportuno de lepra, así como de monitorear y atender los casos con discapacidad grado 1 .

Aunque los resultados del presente análisis se deben valorar a la luz de las limitaciones propias de los estudios retrospectivos, el elevado número de pacientes incluidos en esta investigación y el haberla desarrollado en un centro nacional especializado en la atención de enfermos de lepra le aportan robustez a sus hallazgos y conclusiones.

Se puede concluir que el tiempo transcurrido entre la aparición de los primeros síntomas y el diagnóstico constituye el factor pronóstico clave de la presencia de discapacidades al momento del diagnóstico de lepra. Se observó que mientras mayor es el retraso en el diagnóstico mayor proporción de pacientes son diagnosticados con discapacidad (grados 1 ó $2)$, tanto en mujeres como en hombres. Se debe prestar la mayor atención a prevenir la transmisión de esta enfermedad por ser una importante causa de discapacidad. Se deben monitorear los casos con discapacidad grado 1 ya que, además de posibilitar su reversión, constituyen un indicador temprano del retraso en el diagnóstico.

Se recomienda hacer un seguimiento permanente a los pacientes con lepra del sexo masculino, de 30 años o más, con diagnóstico de lepra del tipo multibacilar e índice bacilar $\geq 2$, especialmente si son residentes de Cundinamarca o Santander. Además de recibir sesiones de fisioterapia más intensas, tanto preventivas como de rehabilitación, y evaluarse con mayor frecuencia (trimestralmente), este grupo de pacientes y sus familiares deben recibir charlas de capacitación sobre la enfermedad y las posibilidades de prevención de la discapacidad, a fin de que se acojan a las estrategias de atención integral que se llevan a cabo en el programa de control.

Se recomienda a las autoridades nacionales y locales de salud establecer una meta de trabajo que lleve a incrementar la proporción de pacientes diagnosticados en menos de un año después de la aparición de los síntomas de lepra, para poder disminuir las altas cargas bacilares iniciales, la discapacidad al momento del diagnóstico y la transmisión y aparición de nuevos casos. Para ello, se debe reforzar la búsqueda activa de personas infectadas y promover el diagnóstico precoz.

Agradecimientos. Los autores agradecen a Juan Carlos Salazar, del Departamento de Historias Clínicas; Claudia Colorado, del Grupo de Apoyo Diagnóstico; Emma López, de la Oficina de Trabajo Social; y Paula Rodríguez, del Consultorio de Fisioterapia, del CDFLLA, por su colaboración en el desarrollo de este trabajo.

Financiamiento. Esta investigación fue totalmente financiada por el CDFLLA bajo el proyecto $4000-16.2 \mathrm{~N}$.

Conflictos de interés. Ninguno declarado por los autores.

\section{REFERENCIAS}

1. World Health Organization, Regional Office for South-East Asia. Enhanced global strategy for further reducing the disease burden due to leprosy. Period 2011-2015. Geneva: WHO; 2009. (SEA-GLP-2009.3).

2. Nicholls PG, Bakirtzief $Z$, van Brakel $W H$, Das-Pattanaya RK, Raju MS, Norman G, et al. Risk factors for participation restriction in leprosy and development of a screening tool to identify individuals at risk. Lepr Rev. 2005;76(4):305-15.

3. Van Brakel WH. Peripheral neuropathy in leprosy and its consequences. Lepr Rev. 2000; 71(Suppl):S146-53.

4. Organización Mundial de la Salud. Estrategia mundial para aliviar la carga de la lepra y sostener las actividades de control de la enfermedad, 2006-2010. Ginebra: OMS; 2005.

5. World Health Organization. Global leprosy situation 2010. Wkly Epidemiol Rec. 2010;85: $337-48$.

6. World Health Organization. Leprosy update, 2011. Wkly Epidemiol Rec. 2011;86:389-400.
7. World Health Organization. Global leprosy situation 2008. Wkly Epidemiol Rec. 2008;83: 293-300.

8. Van Veen NH, Meima A, Richardus JH. The relationship between detection delay and impairment in leprosy control: a comparison of patient cohorts from Bangladesh and Ethiopia. Lepr Rev. 2006;77(4):356-65.

9. Nicholls PG, Croft RP, Richardus JH, Withington SG, Smith WC. Delay in presentation, an indicator for nerve function status at registration and for treatment outcome-the experience of the Bangladesh Acute Nerve Damage Study Cohort. Lepr Rev. 2003;74(4):349-56.

10. Meima A, Saunderson PR, Gebre S, Desta K, van Oortmarssen GJ, Habbema JD. Factors associated with impairments in new leprosy patients: the AMFES cohort. Lepr Rev. 1999;70(2):189-203.

11. Colombia, Ministerio de la Protección Social. Guía 18, guía de atención de la lepra. Bogotá, D.C.: MPS; 2008. Disponible en: http://www. nacer.udea.edu.co/pdf/libros/guiamps/ guias18.pdf Acceso el 3 de junio de 2011.

12. Miranzi SS, Pereira LH, Nunes AA. Perfil epidemiológico da hanseníase em um municipio brasileiro, no período de 2000 a 2006. Rev Soc Bras Med Trop. 2010;43(1):6-27.

13. Moet FJ, Schuring RP, Pahan D, Oskam L, Richardus JH. The prevalence of previously undiagnosed leprosy in the general population of northwest Bangladesh. PLoS Negl Trop Dis. 2008;2:e198.

14. Rodríguez G, González R, González D, Granados C, Pinto R, Herrera $H$, et al Búsqueda activa de lepra y de otras enfermedades de la piel en escolares de Agua de Dios, Colombia. Rev Salud Publica (Bogotá). 2007; 9(3):430-8.

15. Buitrago MT, Casallas AL, Ortiz SP. Perfil sociodemográfico y clínico de los pacientes con lepra atendidos en el Hospital San Salvador de Chiquinquirá, Boyacá, durante el período 1951-1999. Rev Cienc Salud (Bogotá). 2003;1(1):13-30. 
16. De Rijk AJ, Gabre S, Byass P, Berhanu T. Field evaluation of WHO-MDT of fixed duration at ALERT, Ethiopia: the AMFES project-I. MDT course completion, case-holding and another score for disability grading. Lepr Rev. 1994;65(4):305-19.

17. Lubbers WJ, Schipper A, Hogeweg M, De Soldenhoff R. Eye disease in newly diagnosed leprosy patients in eastern Nepal. Lepr Rev. 1994;65(3):231-8.

18. Courtright P, Daniel E, Sundarrao J, Ravanes J, Mengistu F, Belachew M, et al. Eye disease in multibacillary leprosy patients at the time of their leprosy diagnosis: findings from the Longitudinal Study of Ocular Leprosy (LOSOL) in India, the Philippines, and Ethiopia. Lepr Rev. 2002;73(3):225-38.

19. Muvdi S, Muñoz D, Segura MM, Porras de Quintana L. Descripción de las alteraciones oculares en pacientes con enfermedad de Hansen. Médicas UIS. 2006;19(3):157-62.
20. Ramos JM, Reyes F, Lemma D, Belichon I, Gómez JR. Disability profile in leprosy patients' diagnoses in a rural reference leprosy centre in Ethiopia during 1999-2009. Trop Doct. 2011;41(1):51-3

21. Gonçalves SD, Sampaio RF, Antunes CM. Fatores preditivos de incapacidades em pacientes com hanseníase. Rev Saude Publica. 2009:43(2):267-74.

22. Moreira MV, Waldman EA, Martins CL. Hanseníase no estado do Espíritu Santo, Brasil: uma endemia em ascensão? Cad Saude Publica. 2008;24(7):1619-30.

23. Colombia, Ministerio de Salud y Protección Social. Gestión de hospitales públicos [sitio en Internet]. Bogotá, D.C.: Dirección de Prestación de Servicios y Atención Primaria, MSPS; sin fecha. Disponible en: http://201.234.78.38/ SIHO/ Acceso el 3 de junio de 2011.

24. Ferreira J, Mengue S, Wagner M, Duncan B. Estimating hidden prevalence in Hansen's disease through diagnosis delay and grade of disability at time of diagnosis. Int J Leprosy. 2000;68(4):464-72.

25. Cuevas L, De la Hoz F, Guerrero MI, León CI. Utilidad de la estimación de la prevalencia oculta de lepra, basado en el grado de discapacidad al momento del diagnóstico. Colección de Tesis del Postgrado de Salud Pública. Bogotá D.C.: Universidad Nacional de Colombia; 2004.

Manuscrito recibido el 27 de abril de 2012. Aceptado para publicación, tras revisión, el 5 de octubre de 2012

ABSTRACT Objective. Evaluate predictive factors of disability at time of leprosy diagnosis in a cohort of Colombian patients, from 2000 to 2010.

\section{Delay in leprosy diagnosis as a predictor of disability in a cohort of patients in Colombia, 2000-2010}

Methods. Descriptive and analytical observational study of a retrospective cohort of patients admitted with a leprosy diagnosis to the Centro Dermatológico Federico Lleras Acosta in Bogotá, Colombia, from 2000 to 2010. Variables were analyzed descriptively and predictive factors for disability at diagnosis were identified through simple and multifactorial analyses (Cox proportional hazards model); hazard ratios for each factor in the model were calculated.

Results. Time between first symptoms and diagnosis in the 333 cohort patients was 2.9 years on average; $32.3 \%$ had certain degree of disability, especially for the feet. Delay in diagnosis and disability was greater in men than in women and in patients with multibacillary rather than paucibacillary leprosy. Disability was significantly associated with delays of $\geq 1$ year in diagnosis, age $\geq 30$ years, initial bacillary index of $\geq 2$, multibacillary leprosy, and natives of the Cundinamarca or Santander departments. Protective factors were female sex, having some education, and residence in Boyacá.

Conclusions. Time between first symptoms and diagnosis is the key predictive factor of disability at time of leprosy diagnosis. Strengthening of active searching for infected people and promotion of early diagnosis are recommended.

Key words Leprosy; risk factors; delayed diagnosis; disabled persons; Colombia. 